\title{
The Study of Aerodynamic Interactions between Meteoroid Fragments
}

\author{
V.T. Lukashenko, F.A. Maksimov \\ Institute for Computer Aided Design of RAS, Moscow, 123056, Russia \\ lukashenko-vt@yandex.ru,f_a_maximov@mail.ru
}

\begin{abstract}
An algorithm is presented for solving coupled aerodynamic and ballistic problems, based on the simulation method with the use of a grid system and that allows to consider a flight for a system of bodies taking into account deceleration, relative movement of bodies and rotation. The algorithm is used to study the separation of two identical bodies of rectangular shape with different values of the initial moment of inertia. The rotation leads to the fact that the bodies fly apart not only under the action of a repulsive aerodynamic force, but also due to a lifting force appearing because of the body tilt. Additional lateral speed is acquired after the flight section of bodies with significant interference. When rotation is taken into account, the separation velocity of two meteoroid fragments of rectangular shape may increase up to three times in comparison with situation when it is assumed that the bodies do not rotate. The study was carried out with the aim of assessing the influence of various factors on the speed of separation of meteoroid fragments in order to help with construction of possible trajectories of meteorites that fell onto the Earth's surface.
\end{abstract}

Keywords: modeling, meteoroid, fragmentation, interference, rotation.
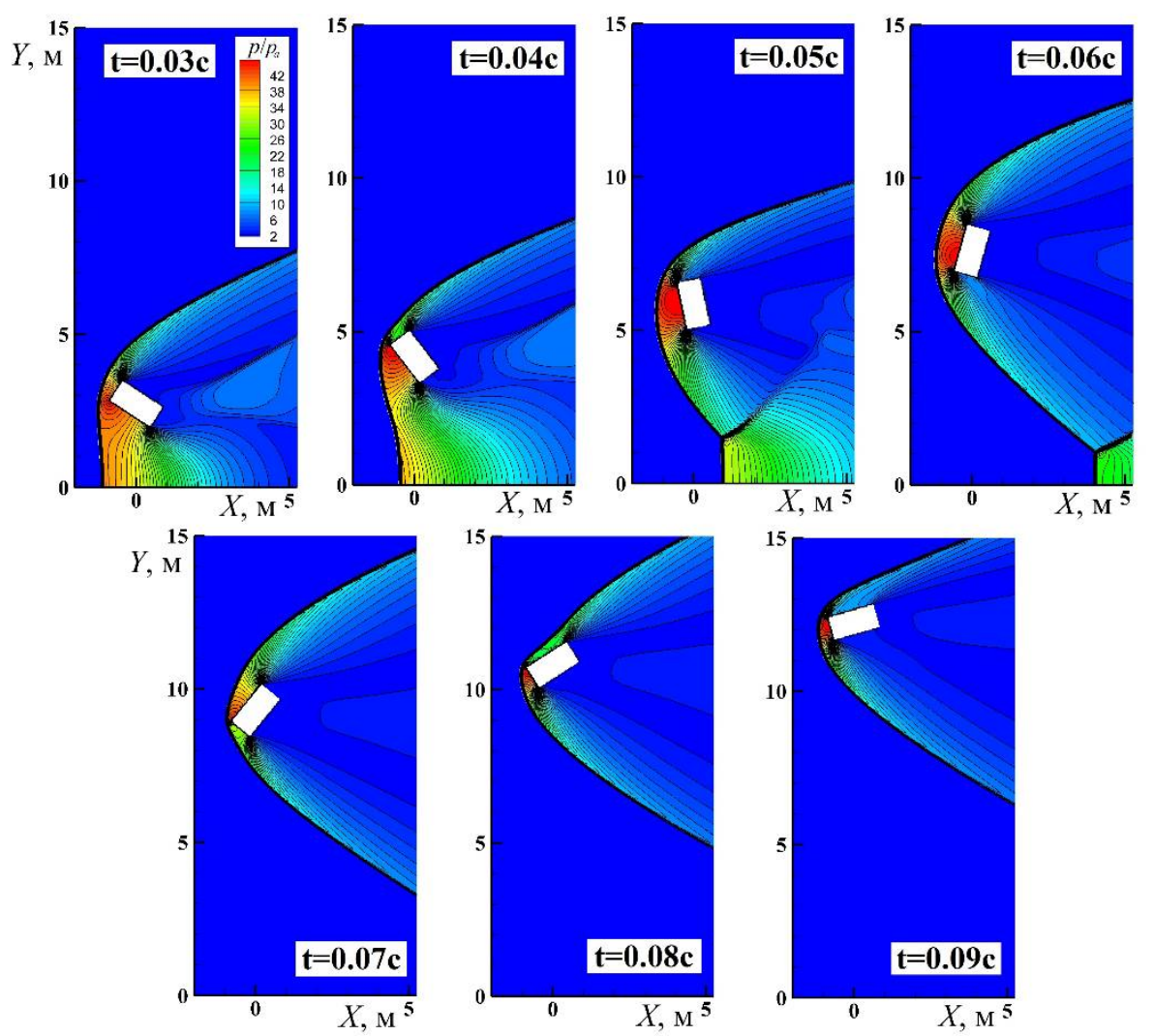

Pictures of the pressure distribution during the separation of two rectangular bodies with an initial angle of attack $\alpha_{0}=+10^{\circ}$ (the upper part of the symmetrical flow pattern) 
УДК 519.6+523.68

\title{
Исследование аэродинамического взаимодействия фрагментов метеорного тела
}

\author{
В.Т. Лукашенко, Ф.А. Максимов \\ Институт автоматизации проектирования РАН, \\ Россия, Москва, 123056, ул. 2-ая Брестская, д.19/18 \\ lukashenko-vt@yandex.ru,f_a_maximov@mail.ru
}

\begin{abstract}
Аннотация
Представлен алгоритм решения сопряженной аэродинамической и баллистической задач, основанный на методе моделирования с использованием системы сеток и позволяющий рассматривать полет системы тел с учетом торможения, относительного перемещения тел и вращения. Алгоритм применен для исследования разлета двух одинаковых тел прямоугольной формы при различных значениях начального момента инерции. Вращение приводит к тому, что тела разлетаются не только под действием расталкивающей аэродинамической силы, но и подъемной силы, возникающей из-за наклона тел. Дополнительная боковая скорость приобретается после участка полета тел со значительной интерференцией. При учете вращения скорость разлета двух осколков метеорного тела прямоугольной формы может увеличиваться до трех раз по сравнению с вариантом, когда предполагается, что тела не вращаются. Исследование проведено с целью оценки влияния различных факторов на скорость разлета осколков метеорного тела после разрушения для построения возможных траекторий выпавших на поверхность Земли метеоритов.
\end{abstract}

Ключевые слова: моделирование, метеорное тело, фрагментация, интерференция, вращение.

\section{1. Введение}

При движении в атмосфере Земли метеорное тело постепенно разрушается под действием увеличивающего скоростного напора и теплового нагрева $[1,2]$. В случае если представленное тело является достаточно массивным, то оно может расколоться на отдельные фрагменты, которые будут продолжать свое движение совместно. Аэродинамические свойства тел в образовавшейся системе фрагментов могут значительно отличаться от аэродинамических свойств тел аналогичной геометрии, но обтекаемых изолированно. Задачу о моделировании динамики таких систем необходимо решать с учетом взаимного влияния тел друг на друга.

В большинстве случаев форма метеорного тела и его фрагментов неизвестна, и, в связи с этим, данные тела представляются как тела малого удлинения, приближенно соответствующие сфере [2]. Для оценки возможного влияния несферической формы на значение коэффициента аэродинамического сопротивления широко используются формы прямоугольного бруса и плиты $[3,4]$. Прямоугольная форма в отличие от сферической обладает моментом тангажа, т.е. при углах атаки на теле прямоугольной формы образуется момент, приводящий к вращению данной формы при полете в атмосфере, однако влияние вращения на полет метеорного тела и разлет его фрагментов обычно не рассматривается $[2,5]$. Для изучения динамики полета системы тел прямоугольной формы с учетом вращения необходимо определить аэродинамические свойства тел не только при различном взаимном положении, но и при разном угле поворота каждого из тел. Большое количество возможных вариантов состояния системы приводит к идее использования сопряженной постановки задачи, когда аэродинамическая и баллистическая задачи решаются параллельно. В этом случае наиболее трудоемкая аэродинамическая задача решается только для тех вариантов состояния системы, которые реализуются 
в полете. Пример исследования динамики двух тел сферической формы с помощью решения сопряженной задачи можно найти в [6]. Исходя из текущей конфигурации, рассматривается задача об обтекании системы, и определяются аэродинамические свойства каждого отдельного тела. Затем скорости и координаты тел изменяются в соответствии с имеющимися скоростями и действующими на тела силами.

В работе [7] авторами был разработан и реализован метод решения сопряженной задачи с учетом относительного перемещения тел. Для моделирования задачи обтекания применяется система сеток [8]. Преимуществом подобного подхода при моделировании является возможность рассматривать обтекание тел различных размеров и форм в достаточно произвольной конфигурации. В [7] алгоритм решения сопряженной задачи применен для исследования задачи о разлете двух одинаковых тел. Произведено тестирование алгоритма на задаче о разлете двух круговых цилиндров. Рассмотрено, как форма тел может оказывать влияние на скорость разлета, получены необходимые коэффициенты для расчета скорости разлета тел эллиптического и прямоугольных сечений в зависимости от их удлинения, однако предполагалось, что тела не вращаются. Целью данной работы является исследование разлета двух тел прямоугольной формы с учетом вращения и оценка влияния вращения на скорость их разлета.

\section{2. Моделирование течения и алгоритм решения сопряженной задачи}

Рассматривается двумерная плоская задача. Для моделирования течения используется система сеток. Для описания внешнего невязкого поля течения используется равномерная по пространству сетка в виде прямоугольных ячеек. На данную сетку, покрывающую всю рассматриваемую область течения, накладывается набор сеток, моделирующих течение около поверхности обтекаемых тел. Данные сетки связаны с поверхностью обтекаемых тел, строятся только в их окрестности, и предназначены для учета формы элементов проницаемой поверхности. Возможность перемещения и вращения сеток, построенных около обтекаемых тел, относительно внешней прямоугольной сетки позволяет производить расчет в сопряженной постановке. Математическая постановка задачи, метод решения уравнений, алгоритм сопряжения решений на перекрывающихся сетках приведены в [7,8].

На подготовительном этапе методом установления рассчитывается поле течения около задаваемой начальной конфигурации системы тел. Учитывается относительное положение тел, но, как и при испытаниях в аэродинамических трубах, не берется во внимание различие в скорости тел. Затем по найденному распределению давления рассчитываются аэродинамические силы и моменты, действующие на каждое отдельное тело.

На следующем шаге решается баллистическая задача. В плоском случае движение центра масс каждого отдельного тела системы описывается системой уравнений

$$
\frac{\mathrm{d} x}{\mathrm{~d} t}=u, \quad m \frac{\mathrm{d} u}{\mathrm{~d} t}=-c_{x} \cdot 0.5 \rho_{a} V^{2} S, \quad \frac{\mathrm{d} y}{\mathrm{~d} t}=v, \quad m \frac{\mathrm{d} v}{\mathrm{~d} t}=c_{y} \cdot 0.5 \rho_{a} V^{2} S,
$$

где $t$ - время; $x, y$ - координаты центра масс; $u, v$ - проекции скорости тела соответственно на направления осей координат $O x$ и $O y ; V$ - величина модуля скорости; $m$ - масса тела; $\rho_{a}$ - плотность воздуха (среды), в которой движется система тел; $S$ - характерная площадь тела; $c_{x}, c_{y}$ - коэффициенты аэродинамического сопротивления и подъемной силы. Вращение тела относительно центра масс описывается уравнениями

$$
\frac{\mathrm{d} \alpha}{\mathrm{d} t}=\omega, \quad I_{z z} \frac{\mathrm{d} \omega}{\mathrm{d} t}=m_{z} \cdot 0.5 \rho V^{2} S L
$$

Здесь $\alpha$ - угол атаки; $\omega$ - угловая скорость вращения тела относительно оси $O z ; I_{z z}-$ момент инерции тела относительно оси $O z ; m_{z}$ - коэффициент момента тангажа; $L$ - характерный размер тела. 
Система уравнений (1), (2) для каждого из тел системы определяет динамику системы в целом. Аэродинамические коэффициенты $c_{x}, c_{y}, m_{z}$, необходимые для ее решения, определяются по распределению давления на поверхности тел из расчета задачи обтекания. Затем производится перерасчет состояния системы (координаты и скорости тел, угол поворота и угловая скорость) через некоторый малый интервал времени $\Delta t$. Аэродинамические коэффициенты при этом определяются из решения газодинамической задачи, а значения баллистических параметров - из текущих значений для каждого обтекаемого тела. После этого тела перемещаются - каждое тело на расстояние, соответствующее относительному смещению данного тела в системе. На шаг интегрирования по времени $\Delta t$ накладываются ограничения: шаг интегрирования не может быть больше задаваемой величины, и при этом максимальное смещение и угол поворота каждого из тел также не должно быть более фиксируемых величин.

После расчета состояния системы на новом временном слое производится перемещение и поворот тел в газодинамической задаче. В соответствии с известным шагом интегрирования динамической задачи производится перерасчет поля течения через данный интервал времени. После интегрирования газодинамической задачи по времени определяется новое распределение давления на поверхности тел, и соответственно значения аэродинамических характеристик тел на новом временном слое. Система готова к повторному интегрированию по времени. Итерационным методом прослеживается динамика системы на больших временных интервалах.

\section{3. Разлет двух тел прямоугольной формы с учетом вращения}

Если рассматривается сверхзвуковой разлет двух одинаковых симметричных тел без учета вращения, то боковая скорость разлета определяется формулой [7]

$$
V_{p}=V \sqrt{\frac{\rho_{a} S L}{m} f\left(y_{0}\right)}, \text { где } f\left(y_{0}\right)=\int_{y_{0}}^{y_{m}} c_{y}(y) \mathrm{d} y
$$

Здесь $V$ - скорость полета тел (в предположении, что $V \gg V_{p}$ ); $m$ - масса тел; $\rho_{a}$ - плотность воздуха; $S, L$ - характерные площадь и размер тел; $y_{0}, y_{m}$ - соответственно начальное расстояние межу телами и расстояние, при котором аэродинамическое взаимодействие тел прекращается. Фактически, чтобы вычислить скорость разлета двух одинаковых симметричных тел без учета вращения, необходимо вычислить коэффициент боковой силы $c_{y}(y)$ в зависимости от расстояния между телами, и при этом расталкивающая сила будет обусловлена интерференцией между телами.

Реальные метеорные тела могут иметь разнообразную форму. Рассмотрим тела прямоугольной формы - в отличие от сферических тел, для тел прямоугольной формы при повороте на угол атаки появляется боковой момент тангажа и дополнительная подъемная сила, которые приводят к вращению тела и дополнительному ускорению в боковом направлении. Для изучения влияния вращения рассмотрим тело прямоугольной формы с удлинением $\lambda=2.0$, имеющее размеры $a=0.794$ м по оси $O x, b=1.588$ м по оси $O y$ и $c=a=0.794$ м по оси $O z$. Объем данного тела составляет примерно $1 \mathrm{~m}^{3}$ с массой 3300 кг при характерной плотности тела ка-

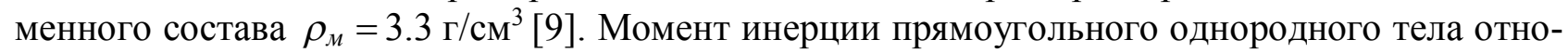
сительно оси $O z$ определяется выражением

$$
I_{z z}=\frac{m\left(a^{2}+b^{2}\right)}{12}
$$

и для данного тела будет составлять $I_{z z}=866 \kappa \Gamma^{*} \mathrm{M}^{2}$.

Изучим разлет двух подобных тел, первоначально расположенных на расстоянии между центрами масс, равном $1.5 \mathrm{a}$. Все расчеты выполнялись в условиях полета со скоростью 15 км/с на высоте 20 км. Параметры атмосферы брались по таблице стандартной атмосферы 
(ГОСТ 4401-81). На рис.1 при трех вариантах относительного расположения тел приведены соответствущие начальные поля течения. Варианты отличаются согласованным поворотом тел (не нарушающим симметрию) относительно оси $O z$ на углы $\alpha=-10,0$ и $+10^{\circ}$. Поворот тел по углу атаки имитирует возмущение по начальной угловой скорости вращения. Газодинамические расчеты проводились при числе Маха $\mathrm{M}=6$, так как аэродинамические свойства тел в рамках теории течений с большой сверхзвуковой скоростью не сильно изменяются при больших числа Маха [10].
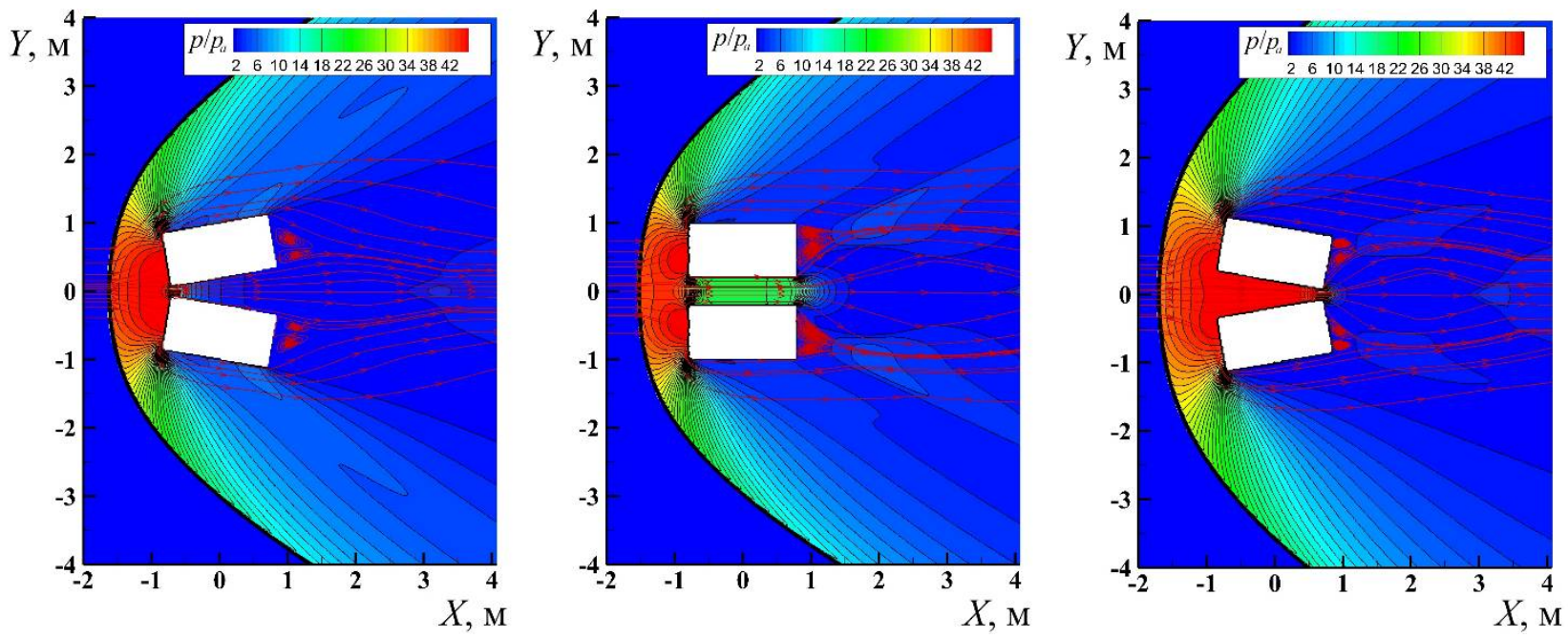

Рис. 1. Распределение давления и линии тока около двух тел прямоугольной формы соответственно при повороте на угол атаки $\alpha=-10,0$ и $+10^{\circ}$

Проведены расчеты сопряженной аэродинамической и баллистической задач по представленному алгоритму. На рис. 2 приведены боковая скорость $V_{p}$ и угол атаки $\alpha$ в зависимости от времени для трех вариантов начального поворота тел. Для случая $\alpha_{0}=0^{\circ}$ малое значение угла атаки сохраняется на протяжении всего времени разлета тел, при этом боковая скорость фактически набирается только из-за интерференции между двумя телами. При удалении друг от друга на достаточное расстояние боковая скорость выходит на константу.

a)
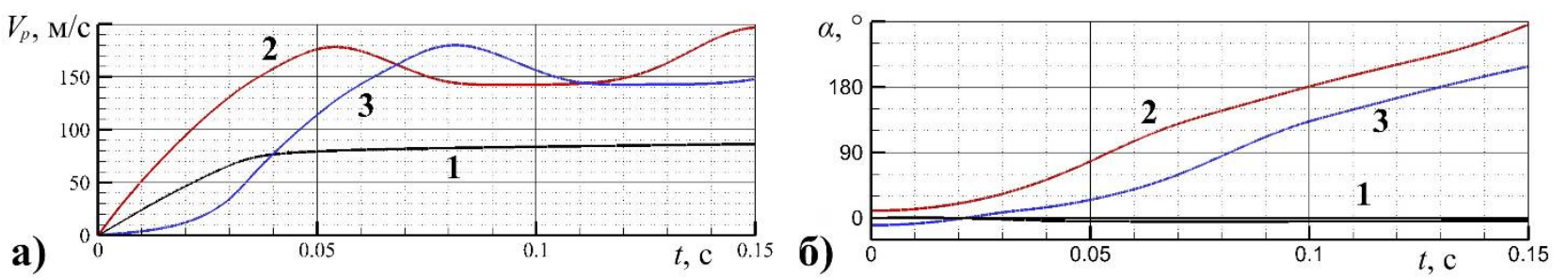

Рис. 2. Боковая скорость (а) и угол атаки (б) при разлете двух тел прямоугольной формы при различных значениях начального угла атаки: $1-\alpha_{0}=0^{\circ} ; 2-\alpha_{0}=+10^{\circ} ; 3-\alpha_{0}=-10^{\circ}$

При достаточном отклонении от состояния аэродинамического равновесия (что в данном случае имитируется наличием начального угла атаки $\alpha_{0}= \pm 10^{\circ}$ ) удлиненное тело начинает вращаться. На рис. 3 представлены последовательные картины течения для моментов времени $t=0.03,0.04,0.05,0.06,0.07,0.08$ и 0.09 с при полете тел с начальным углом атаки $\alpha_{0}=+10^{\circ}$ (приведена половина симметричной картины течения). При этом в обоих вариантах $\left(\alpha_{0}=-10^{\circ}\right.$, $\alpha_{0}=+10^{\circ}$ ) реализуется рост скорости разлета приблизительно в 2 раза (см. рис. 2), связанный с наличием боковой силы, возникающей при выходе тел на угол атаки. Фактически, при разлете двух прямоугольных тел реализуется три режима. Первый режим реализуется при достаточно малом расстоянии между телами - при значительной интерференции боковая сила в ос- 
новном определяется наличием близко расположенного тела. Тела при этом не только разлетаются, но и начинают вращаться. Второй режим реализуется при достаточном удалении тел друг от друга и наличии угла атаки (больше $0^{\circ}$ и меньше $90^{\circ}$ ) - образуется дополнительная боковая сила. Тела при этом продолжают вращаться до возможного аэродинамически устойчивого положения, реализующегося при углах атаки $0,90,180^{\circ}$ и т.д. В дальнейшем на третьем режиме полета двух вращающихся тел без взаимного взаимодействия, создаваемая дополнительная боковая скорость из-за вращения будет в среднем равна 0. В приведенных примерах первый режим длится приблизительно $0.04 \mathrm{c}$, а второй режим заканчивается на временном интервале $0.06 \div 0.08$ c.
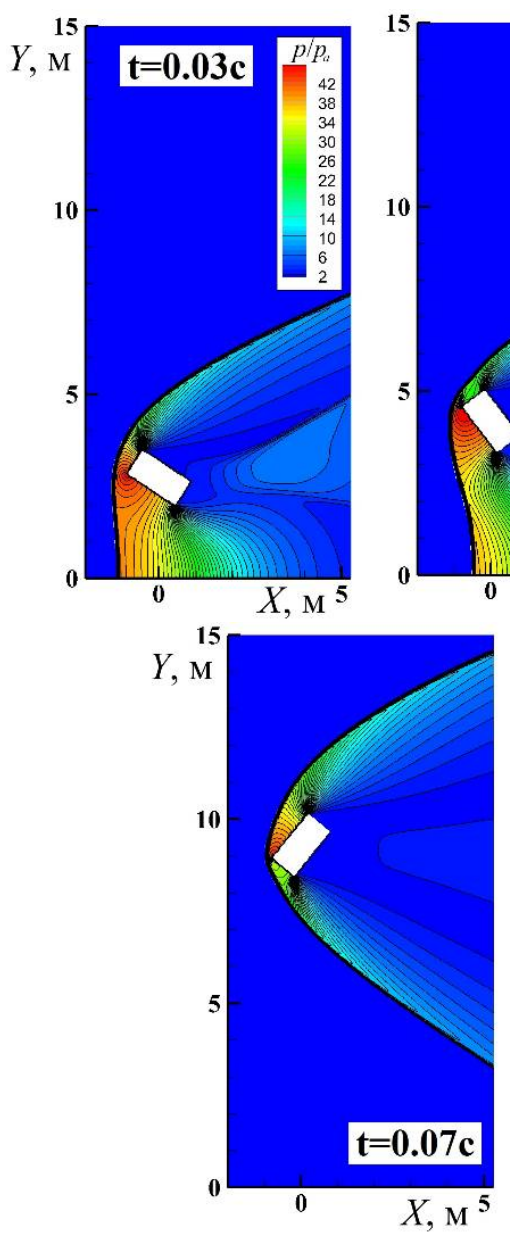
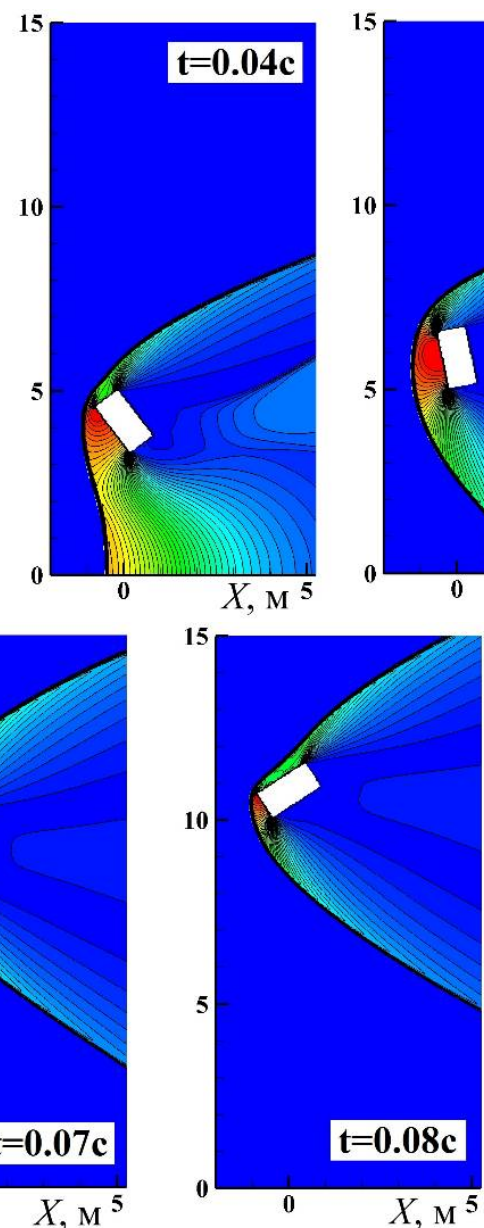
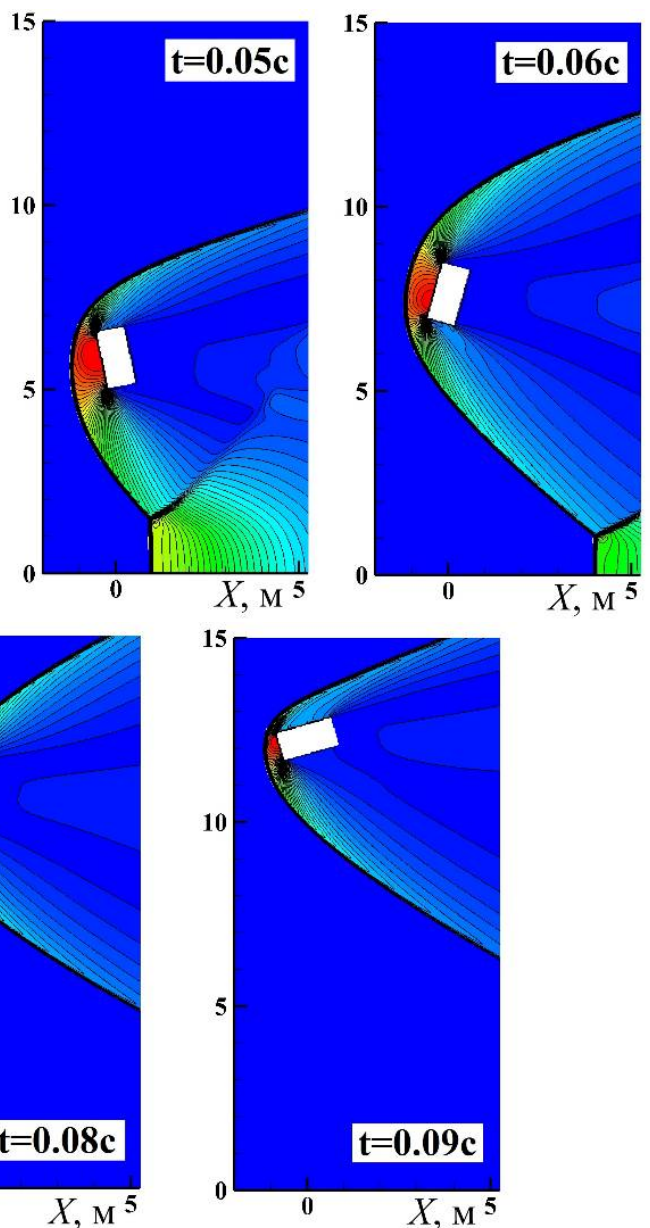

Рис. 3. Картины распределения давления при разлете двух прямоугольных тел с начальным углом атаки $\alpha_{0}=+10^{\circ}$

Для определения скорости разлета вращающихся тел важна продолжительность полета во втором режиме. Для того чтобы продемонстрировать это, были выполнены расчеты динамики полета тел с измененным моментом инерции $I_{z z}$. В соответствии с уравнением (2) уменьшение момента инерции приводит к увеличению углового ускорения, а его увеличение - к уменьшению углового ускорения. Следовательно, при уменьшении $I_{z z}$ полетный угол атаки $90^{\circ}$ будет достигаться раньше. А при увеличении - позже. Таким образом, можно изменять время полета тел во втором режиме.

На рис. 4 приведены боковая скорость $V_{p}$ (рис. 4, а) и угол атаки $\alpha$ (рис. 4,6 ) в зависимости от времени для шести вариантов расчетов с моментом инерции $I_{z z}=k I_{z z 0}$, где $I_{z z 0}=866 \kappa^{\circ} \mathrm{M}^{2} ; k=0.1,0.25,0.5,1.0,1.5,2.0$. Исследовался вариант с начальным углом атаки $\alpha_{0}=+10^{\circ}$. 
Максимальная боковая скорость увеличивается с ростом момента инерции. При $k=0.1$ тело разворачивается на угол атаки $90^{\circ}$ фактически на первом режиме полета со значительной интерференцией. Максимальная боковая скорость при этом практически такая же, как и для случая полета тела без вращения. То есть, второй режим разлета двух тел в данном случае отсутствует. При $k=0.1$ и 0.25 тела поворачиваются на угол атаки $90^{\circ}$ и данное положение являтся устойчивым положением при дальнейшем полете. При $k=0.5$ набираемая угловая скорость достаточна для вращения до угла атаки $180^{\circ}$ и более. При $k=1.5$ и 2.0 продолжительность полета во втором режиме с "эффективным" (для роста скорости разлета) углом атаки между телами увеличивается, что приводит к дополнительному росту боковой скорости.

a)

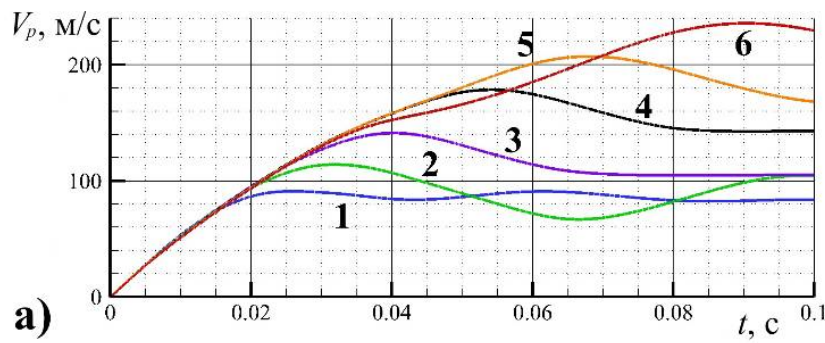

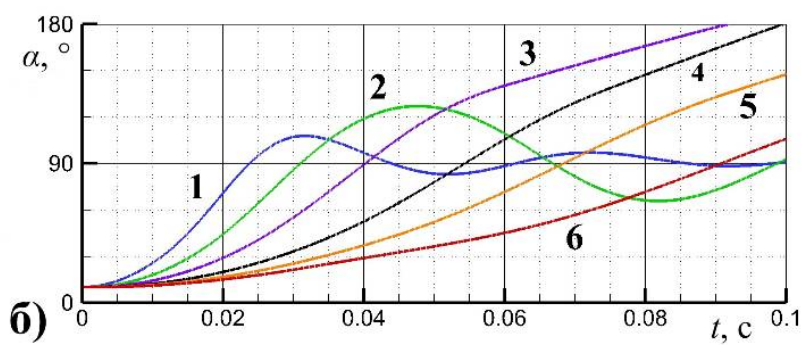

Рис. 4. Изменение боковой скорости (а) и угла атаки (б) при моменте инерции $I_{z z}=k I_{z z 0}$ : $1-k=0.1 ; 2-k=0.25 ; 3-k=0.5 ; 4-k=1.0 ; 5-k=1.5 ; 6-k=2.0$

При отсутствии вращения или очень быстром вращении тел скорость разлета тел составляет $80 \div 90 \mathrm{M} /$ c. При учете вращения максимальная боковая скорость для рассмотренных вариантов расположения тел увеличивается до $180 \mathrm{~m} / \mathrm{c}$, а при увеличении момента инерции - до 240 м/с. Увеличение момента инерции реального тела может быть обусловлено неоднородным распределением массы или большим удлинением геометрической формы при рассмотрении однородных тел, однако в этом случае необходимо также учитывать изменение аэродинамических свойств.

На рис. 5 приведены аэродинамические характеристики прямоугольного тела с удлинением $\lambda=2.0$ при числе Маха $\mathrm{M}=6$ в зависимости от угла атаки (от 0 до 90 $)$ при изолированном обтекании (линиия 1 - темная с крупными маркерами) и при наличии симметрично расположенного аналогичного тела относительно горизонтальной линии на расстоянии между центрами масс, равном 2.0, 3.0, 4.0, 6.0 и 8.0 (соответственно линии 2, 3, 4, 6 и 8). При угле атаки $\alpha=0^{\circ}$ тело расположено длинной стороной вдоль направления движения. За характерную площадь принята площадь миделя За характерный размер длины принята короткая сторона прямоугольного тела. при угле атаки $\alpha=0^{\circ}$.
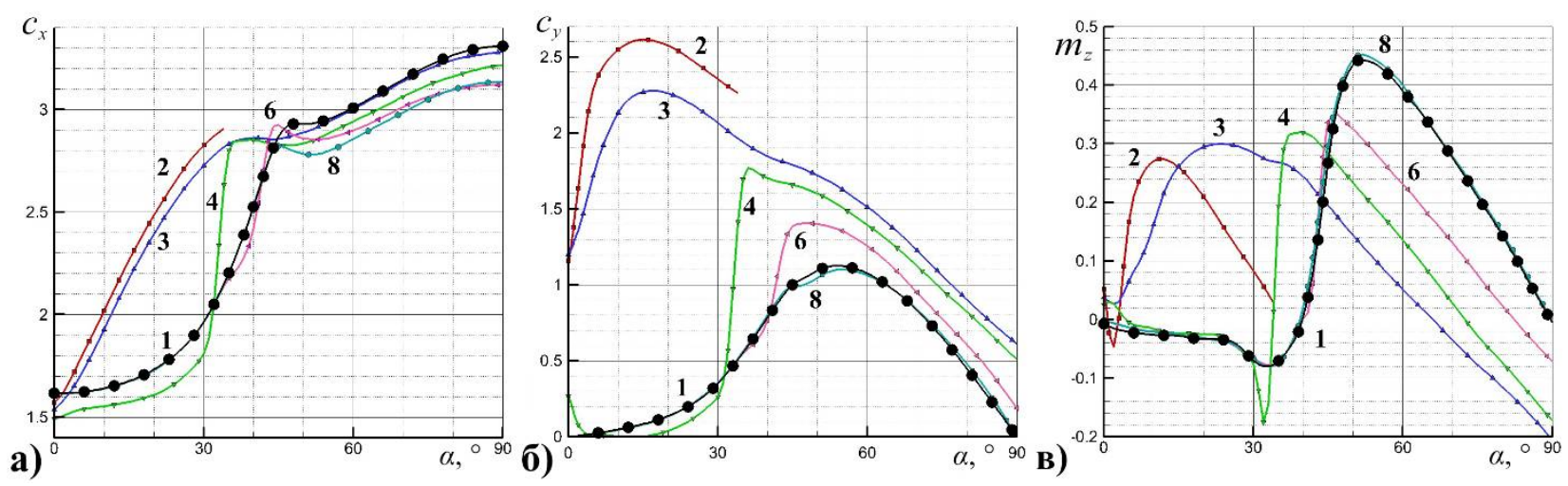

Рис. 5. АДХ прямоугольного тела с удлинением $\lambda=2.0: c_{x}$ (a), $c_{y}$ (б) и $m_{z}$ (в) в зависимости от угла атаки при изолированном обтекании (линия 1) и при наличии симметрично расположенного аналогичного тела на расстоянии, равном 2, 3, 4, 6 и 8 (соответственно линии 2, 3, 4, 6 и 8) 
При расстоянии между центрами масс, равном 2, максимальный угол атаки ограничен из геометрических соображений. При расстоянии между центрами масс меньше 4.0 присутствует значительная интерференция, что приводит к значительному росту подъемной силы, а также коэффициента аэродинамического сопротивления. Отдельно необходимо отметить, что момент тангажа в этих условиях изменяет знак с отрицательного на положительный - это означает, что появляется момент на увеличение угла атаки. При расстоянии между центрами масс более 4.0 тела взаимодействуют только при повороте на достаточно большой угол атаки, при этом аэродинамические силы имеют характеристики, аналогичные обтеканию изолированного прямоугольного тела.

Полученные аэродинамические коэффициенты показывают, что вращение и выход тел на большие углы атаки при начальном режиме со значительной интерференцией не оказывают значительного влияния на скорость разлета двух частей метеорного тела. Однако, если после окончания участка полета со значительной интерференцией, тело оказывается на угле атаки меньше $90^{\circ}$, то боковая скорость может значительно увеличиться (до нескольких раз). Данный участок полета может быть тем больше, чем больше момент инерции тела.

\section{4. Заключение}

Представлен метод решения сопряженной аэродинамической и баллистической задач для исследования обтекания систем тел в условиях значительной интерференции с учетом торможения, боковой силы и момента тангажа. Проведены расчеты динамики разлета тел прямоугольной формы с учетом возможности вращения тела по углу тангажа при различных значениях момента инерции. При разлете двух тел прямоугольной формы кроме расталкивающей силы, из-за вращения образуется дополнительная боковая сила, которая приводит к значительному росту скорости разлета: до трех раз для выполненных расчетов. Реализуется три режима разлета: 1) режим, обусловленный значительной интерференцией между телами, вращение на данном этапе не оказывает значительного влияния на скорость разлета; 2) удаление тел друг от друга при наличии угла атаки, приводящего к вращению тел - в данном режиме тела могут приобретать дополнительную боковую скорость; 3) выход тел на независимое обтекание в аэродинамически устойчивом положении. Продолжительность второго режима разлета тел увеличивается с ростом момента инерции. Результаты расчетов показывают, что вращение и форма тел могут существенно влиять на скорость разлета осколков метеорного тела и, как следствие, приводить к большей площади выпадения метеоритов.

\section{Литература}

1. Григорян С.С. О движении и разрушении метеоритов в атмосфере планет // Космические исследования. 1979. Т. 17, № 6. С. 875-893.

2. Стулов В.П., Мирский В.Н., Вислый А.И. Аэродинамика болидов. М.: Наука. Физматлит, 1995. $240 \mathrm{c}$.

3. Ждан И.А., Стулов В.П., Стулов П.В., Турчак Л.И. Коэффициенты сопротивления тел метеорных форм // Астрономический вестник. 2007. Т. 41, № 6. С. 544-547.

4. Грицевич М.И. Оценка конечной массы крупных метеорных тел// Доклады Академии наук. 2008. T. 423, № 1. С. 44-51.

5. Сызранова Н.Г., Андрущенко В.А. Моделирование движения и разрушения болидов в атмосфере Земли // Теплофизика высоких температур. 2016. Т.54, № 3. С. 328-335.

6. Барри Н.Г. Динамика двух сферических объектов в сверхзвуковом потоке // Доклады Академии Наук. 2010. Т. 434, № 5. С.620-621.

7. Лукашенко В.Т., Максимов Ф.А. Математическая модель разлета осколков метеорного тела после разрушения // Инженерный журнал: наука и инновации. 2017. Вып. 9(69). С. 1-14. 
8. Максимов Ф.А. Сверхзвуковое обтекание системы тел // Компьютерные исследования и моделирование. 2013. Т. 5, № 6. С. 969-980.

9. Britt D.T., Yeomans D. ,Housen K., Consolmagno G. Asteroid Density, porosity and structure // Asteroids III / EdsBottke V.F., Celino A., Paolicchi P.P., Binzel R.P. Tucson: Univ. Arizona Press. 2002. Pp. $485-500$.

10. Черный Г.Г. Течения газа с большой сверхзвуковой скоростью. М.: Физматлит, 1959. 220 с.

Статья поступила в редакцию 14 октября 2019 г. 